\title{
IL TERREMOTO DELLO HOKKAIDO DEL 4 MARZO 1952
}

\author{
Antonino Girlanda
}

\section{Parte III}

Il carattere particolare degli inizi delle $P_{n}$ osservati in un gran numero di registrazioni, la presenza di chiari esempi di tipi di onde che si riscontrano in modo particolare nelle registrazioni di terremoti con ipocentro a profondita superiore alla normale, mi hanno indotto, nellintraprendere il calcolo delle costanti ipocentrali $\left({ }^{1}\right)$ del terremoto dello Hokkaido del 4 marzo 1952, ad attribuire inizialmente una certa profonditi all'ipocentro. Il calcolo, condotto seguendo un metodo statistico di Caloi, ha, tuttavia, escluso la possibilita di una profondita apprezabile, dando luogo ai seguenti risultati:

$$
\begin{aligned}
& \varkappa_{0}=+143^{\circ} 30^{\prime} 00^{\prime \prime} \cdot 3 \pm 03^{\prime} 35^{\prime \prime} \cdot 4, \\
& \text { 小 }=+42^{\circ} 14^{\prime} 38^{\prime \prime} \cdot 8+04^{\prime} 19^{\prime \prime} \cdot 5 \text { (latitudine geocentrica), } \\
& h=0, \\
& \iota_{0}=01^{\mathrm{H}} 22^{m} 41^{\sharp} \cdot 5 \pm 0^{\circ} \cdot 3 .
\end{aligned}
$$

Questo fatto $\dot{e}$ stato successivamente confermato $\left({ }^{2}\right)$ mediante il calcolo della equazione della dromocrona più probabile delle $P_{n}$, che, per un gruppo di 45 stazioni di osservazione ha dato luogo a tempi di tragitto notevolmente coincidenti con gli analoghi tempi dedotti dai valori riportati nella colonna "surface" delle tabelle di Jeffreys e Bullen ( ${ }^{3}$ ). Dal confronto (tabella I) appare evidente come le diffe. renze 0 - $C$, dedotte dalla dromocrona sperimentale (colonna 6), siano sensibilmente coincidenti con le analoghe differenze (colonna 7) de. dotte dalla dromocrona di Jeffreys e Bullen relativa a terremoti superficiali.

Esaminando la tabella I si nota che, mentre per un gruppo di 45 stazioni le differenze $0-C$ si distribuiscono intorno al valore zero con uno scarto medio di $0^{*} .13$, per un gruppo di 14 stazioni (Ambulong, Seattle, Kodaikanal, Szeged, Kalcksa, De Bilt, Pavia, Seven Falls, Wellington, Tortosa, Cartuja, Lisbona, Malaga, Bermuda) tali differen- 


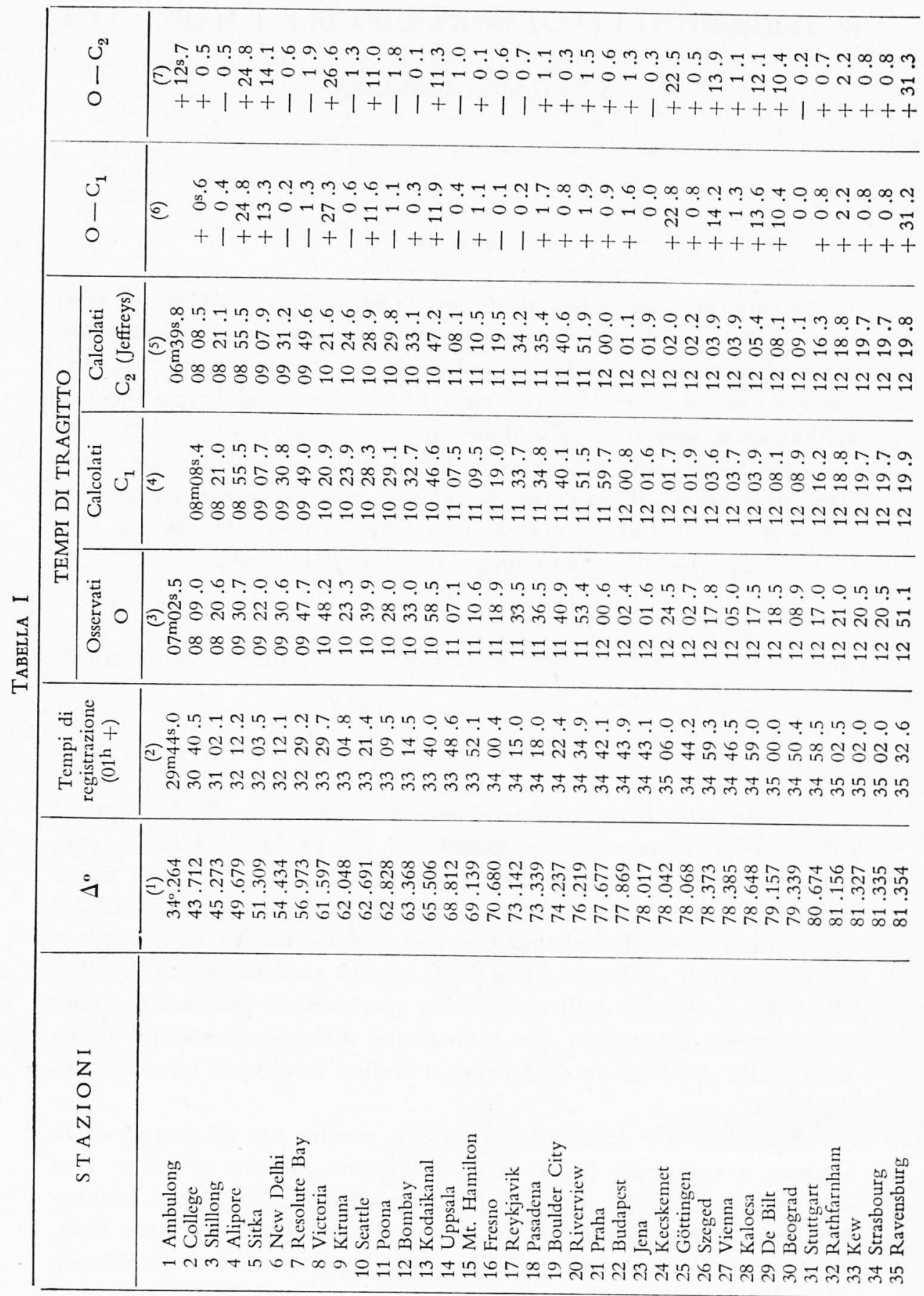




\begin{tabular}{|c|c|}
\hline$j_{0}^{j^{2}}$ & 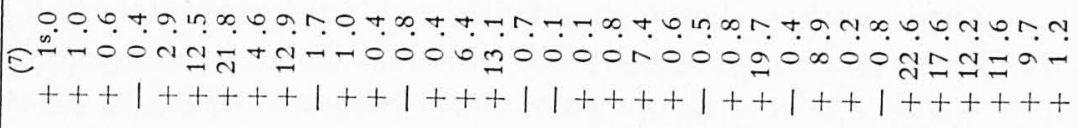 \\
\hline $0_{0}^{-1}$ & 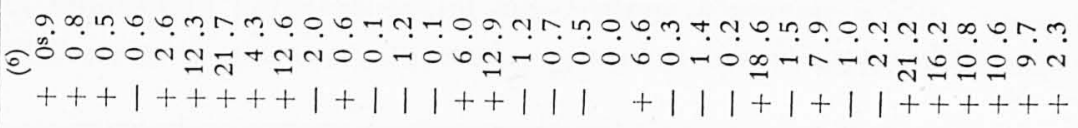 \\
\hline
\end{tabular}

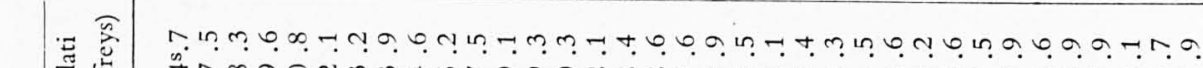
० 品

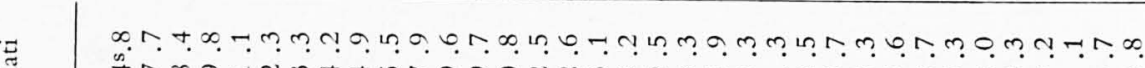

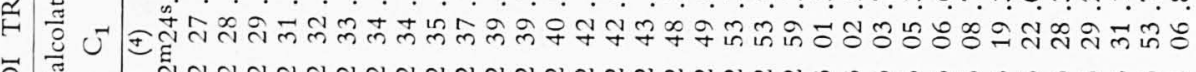
|ื

E In

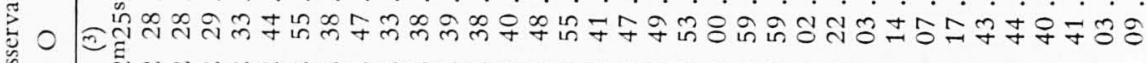
ถ์

n.

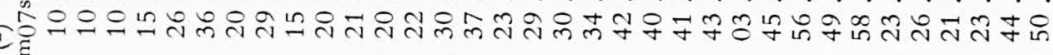

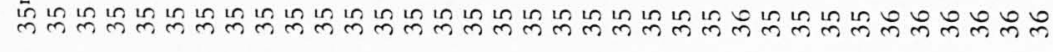

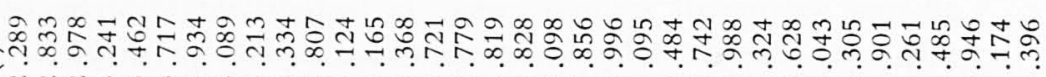

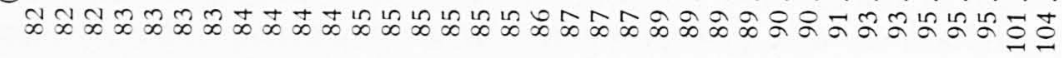

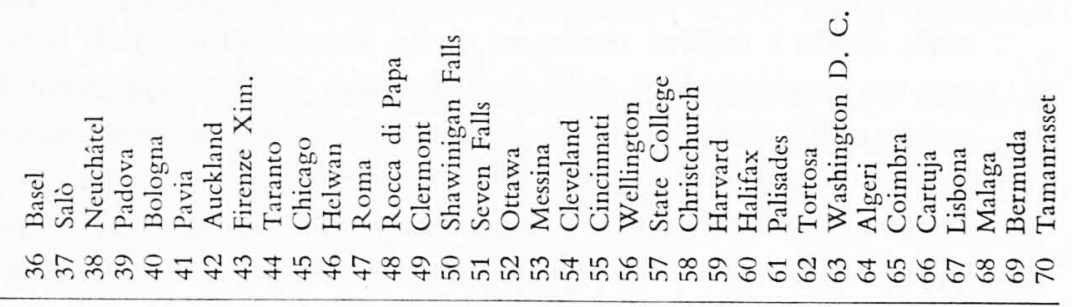


ze si distribuiscono intorno al valore $+11^{*} .6+0.2$. Per tradure in forma grafica questa circostanza, su carta quadrettata si è indicato ogni residuo (arrotondato al secondo) con un cerchietto. Nella fig. 1 appare evidente che, a parte pochi e sparsi residui, si ha un significativo addensamento nell'intorno di 0 " e nell'intorno di $12^{*}$.

Si è osservato, inoltre, che le registrazioni, nonostante la eccezionale intensita del terremoto, iniziano con una lieve emersio seguita,

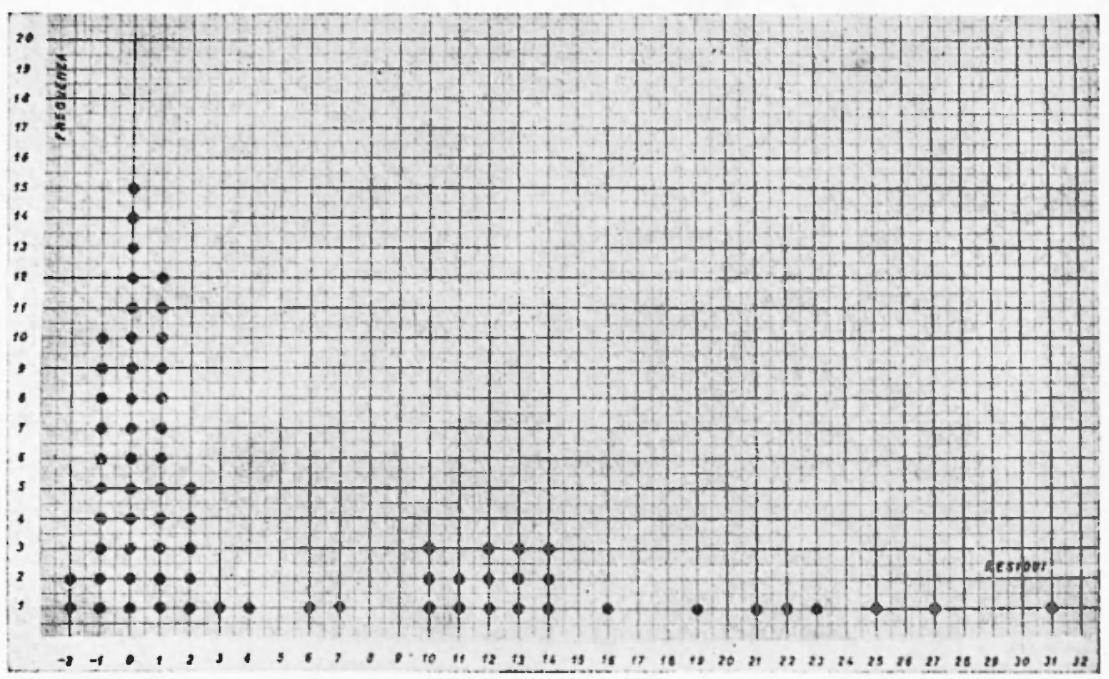

Fig. 1

ad una diecina di secondi circa, da bruschi impulsi che conferiscono alla parte iniziale delle registrazioni un aspetto particolare. Tale aspetto, in alcuni sismogrammi, è talmente mareato che pui far pensare alla registrazione successiva di scosse distinte.

Un riesame dei sismogrammi ha permesso di ilevare che un notevole numero di stazioni presenta una $i\left(P\right.$. circa $12^{*}$ dopo la revistrazione della $P\left(P_{1}\right)$, che generalmente inizia con una 2 . I risultati di queste letture addizionali sono riportati nella tabella ll. Nella fiø. 2, nella quale i residui risultanti dalle letture addizionali sono indicati con un quadratino, si nota con magriore evidenza, a parte l'addensamento nell'intorno di 0 , un più fitto addensamento nell'intorno di $12^{\text {s. }}$.

Circa possilsili errori di identificazione, si può osservare che la f́ase indicata con $P_{2}$ non è identificabile né con la $P P$. che portereblue ad una differenza dell'ordine osservato solo per stazioni con distanza 
epicentrale nell" intorno di 15 ", e nessuna delle stazioni utilizzate cade in tale intorno, né con la PcP che, oltre a non essere solitamente di così facile registrazione, dovrel, le essere distanziata dalla $P$, conformemente ai tempi di tragitto calcolati da Jeffreys e Bullen, di $45^{\mathrm{s}} .9$ per $\Delta=600^{\circ}$, di $22^{\circ} .4$ per $\Delta=70^{\circ}$, di $7^{\circ} .9$ per $\Delta=80^{\circ}$ e di appena $1^{*} .5$ per $\lrcorner=90^{\circ}$. La $P$, potrel,le essere identificata con la $p P$, poiché una differenza dell'ordine osservato è grossolanamente attrilıuilıile alla

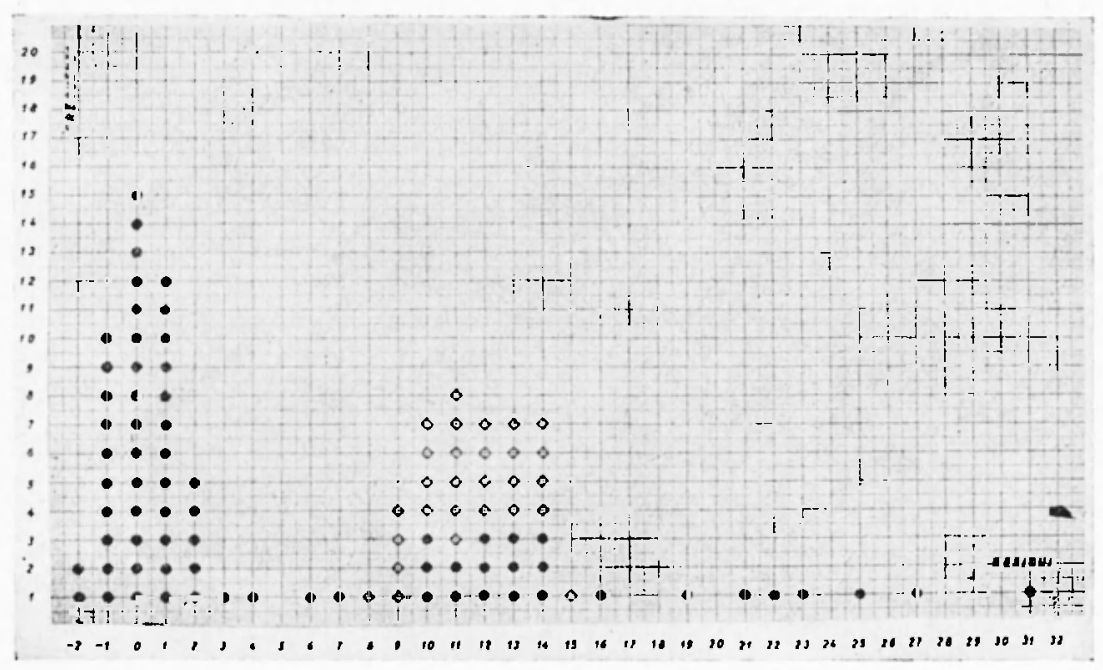

Fig. 2

differenza $p P \ldots P$ per una profondità compresa tra i 60 e i $100 \mathrm{~km}$. Difficilmente, pero, la $P$.. osservata è identificalsile con una $p P$, in quanto. anche volendo prescindere dalle conclusioni stabilite in preceden. za, un osscrvatore che alblia una sufficiente pratica di sismogrammi, difficilmente pui credere che l'inizio, teneralmente netto e vistoso delle $I_{2}$, sia imputabile alla $P P$, specie quando l'inizio delle $P$ è cosi fievole, ne potrelibe spiegarsi sufficientemente il fatto che il gruppo delie it - Lazioni su menzionate alblia potuto registrare le $p P$ senza registrare ie $P$.

Un insieme di fatti analoghi riscontrati nellesaminare i tempi di registrazione delle $P$ elencati nell'International Seismological Summary relativi a due terremoti del Messico del 3 Giugno $1932\left(t^{\prime \prime}=10^{\mathrm{h}} 36^{\mathrm{m}} 53^{\mathrm{s}}\right)$ e del 18 Giugno $1932 \quad\left(t_{0}=10^{\mathrm{h}} 12^{\mathrm{m}} 15^{\mathrm{s}}\right.$, aventi lo stesso epicentro $19^{\prime \prime} .2 \mathrm{~N} . .104^{\prime \prime} .2 \mathbb{W}$. hanno indotto Stoneley (') a considerare entram- 
Tabella II

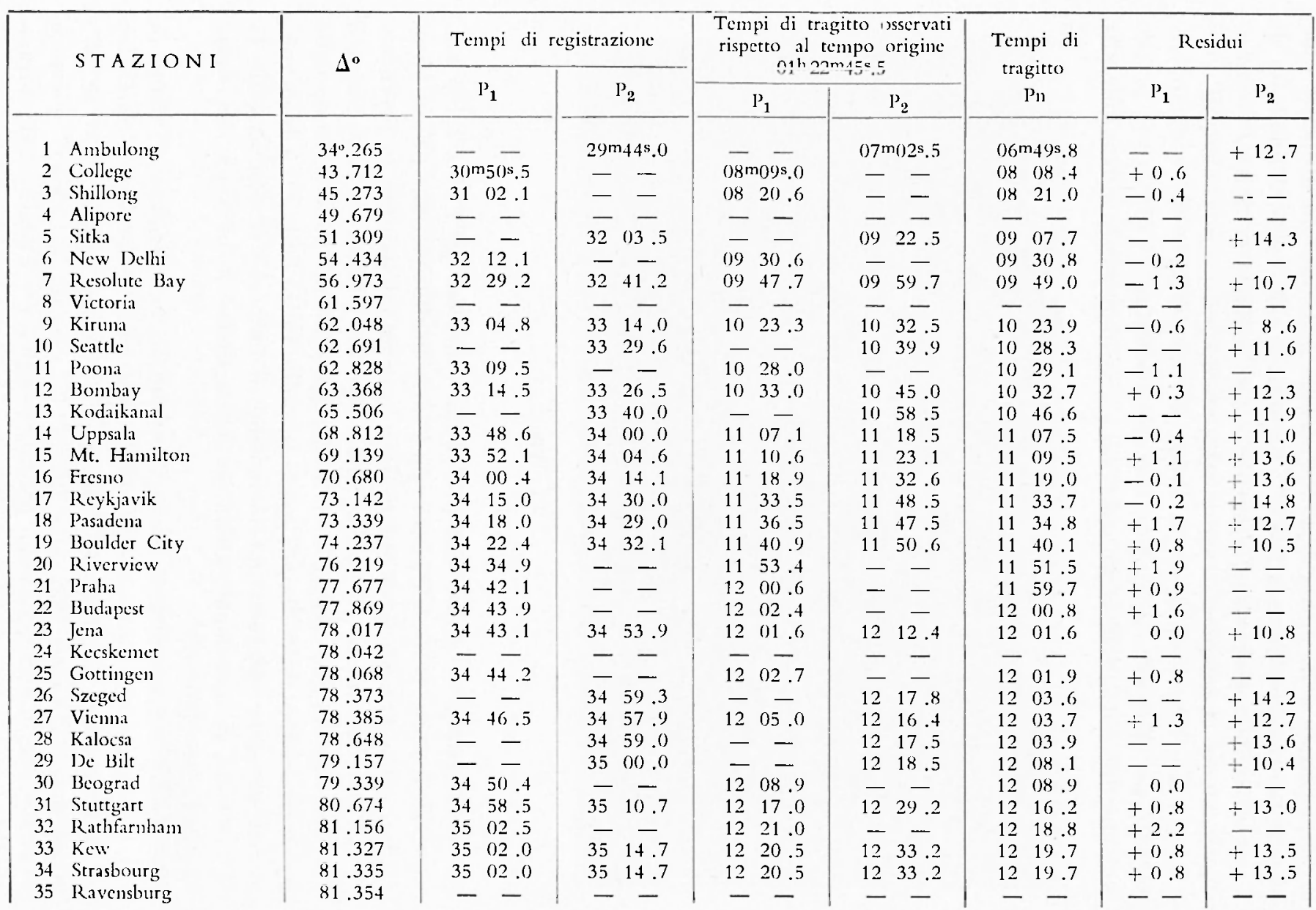


Segue tabella II

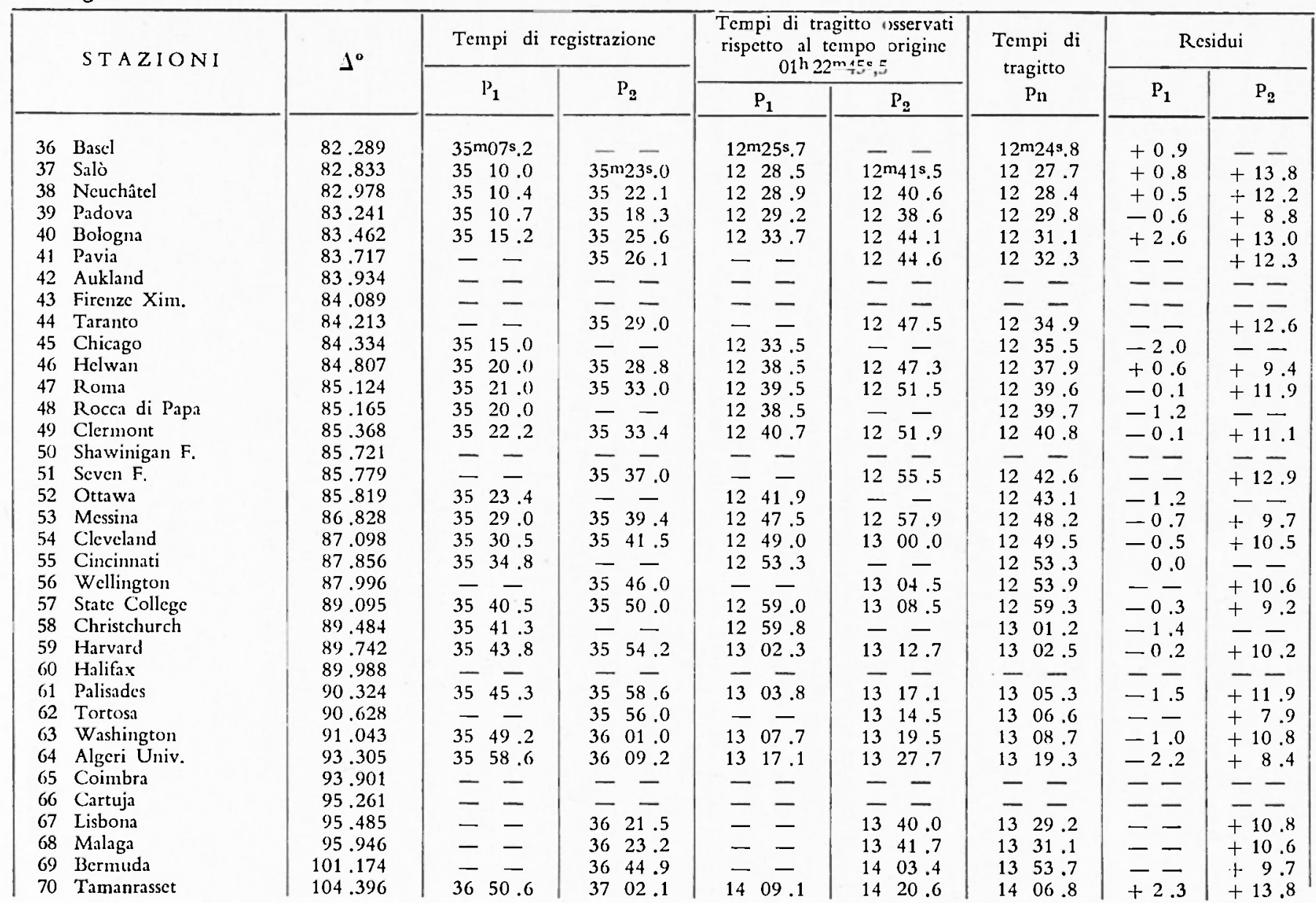


hi i terremoti come doppi, essendo, in ambedue i casi, una scossa dehole seguita da una più forte $19-20$ secondi più tardi. L'analisi dei re. sidui, integrata dallesame diretto dei sismogrammi della slazione di Kew, non hanno fornito allo Stoneley elementi sufficienti per potere stabilire, in entrambi i casi, se le due scosse successive abluino avuto origine in diversi epicentri e a diverse profonditi ipocentrali.

Le considerazioni su riferite, derivate da misure escguite direttamente sui numerosi sismogrammi originali o copie fotografiche in grandezza oriwinale gentilmente inviati da numerosi Osservatori, costituiscono delle prove sufficienti sul carattere multiplo del terremoto dello Hokkaido del + Marzo 1952.

Sorge naturaluente la domanda se, supposta la registrazione sulressiva di due scosse distinte, tali scosse abliano avuto oriqine nello stesso ipocentro o se la prima scossa delle $1^{\mathrm{h}} 22^{\mathrm{m}} 45^{\mathrm{s}} .5$ ne abhia stimolato una seconda ad una certa distanza nello spazio e nel tempo.

Ritenendo la questione di un certo interesse, ho creduto utile procedere alla deteminazione dell̈̈pocentro e del tempo origine della seconda scossa con i dati delle stazioni riportati nella tabella Ill, nella quale, oltre i dati rilevati dalle registrazioni che iniziano con le $P_{2}$ cono inclusi i dati delle registrazioni che iniziano con le $P_{i}$ e contenærono nettamente le $P_{\underline{o}}$.

Per un criterio di uniformiti ho fatto uso dello stesso metodo di Caloi $(\cdots)$ impiegato in precedenza per la determinazione delle costanti ipocentrali della prima scossa (1), che, com” noto. porta alla soluzione di un sistema di $n$ ( $n$ è il numero delle stazioni utilizzate) equazioni del tipo

$$
\delta t_{\mathrm{o}}+b_{\mathrm{i}} \delta \lambda_{\mathrm{o}}+c_{\mathrm{i}} \delta \phi_{\mathrm{o}}+d_{\mathrm{i}} \delta h+l_{\mathrm{i}}=0 \quad(i=1,2, \ldots, n)
$$

nelle quattro incognite $\delta t_{0}, \delta \lambda_{0}, \delta \phi_{0}, \delta / h$, correzioni da apportare a certi valori approssimati $\left(t_{0}\right),\left(\lambda_{0}\right),\left(o_{0}\right),(h)$ del tempo origine, della longitudine, latitudine e profonditi, che si presuppongono noti.

I coefficienti delle [1] hanno le seguenti espressioni :

$$
\begin{gathered}
b_{\mathrm{i}}=\frac{\partial f_{\mathrm{i}}}{\partial\left(\Delta_{\mathrm{i}}\right)} \frac{\partial\left(\Delta_{\mathrm{i}}\right)}{\partial\left(\lambda_{\mathrm{o}}\right)}, \quad c_{\mathrm{i}}=\frac{\partial f_{\mathrm{i}}}{\partial\left(\Delta_{\mathrm{i}}\right)} \frac{\partial\left(\Delta_{\mathrm{i}}\right)}{\partial\left(\omega_{\mathrm{o}}\right)}, \\
d_{\mathrm{i}}=\frac{\partial f_{\mathrm{i}}}{\partial(h)}, \quad l_{\mathrm{i}}=-\left\{T_{\mathrm{i}}-\left[\left(t_{\mathrm{o}}\right)+f_{\mathrm{i}}\left(\left(\lambda_{\mathrm{o}}\right),\left(\Phi_{\mathrm{o}}\right),(h)\right)\right]\right\}
\end{gathered}
$$

col noto significato dei simboli. 


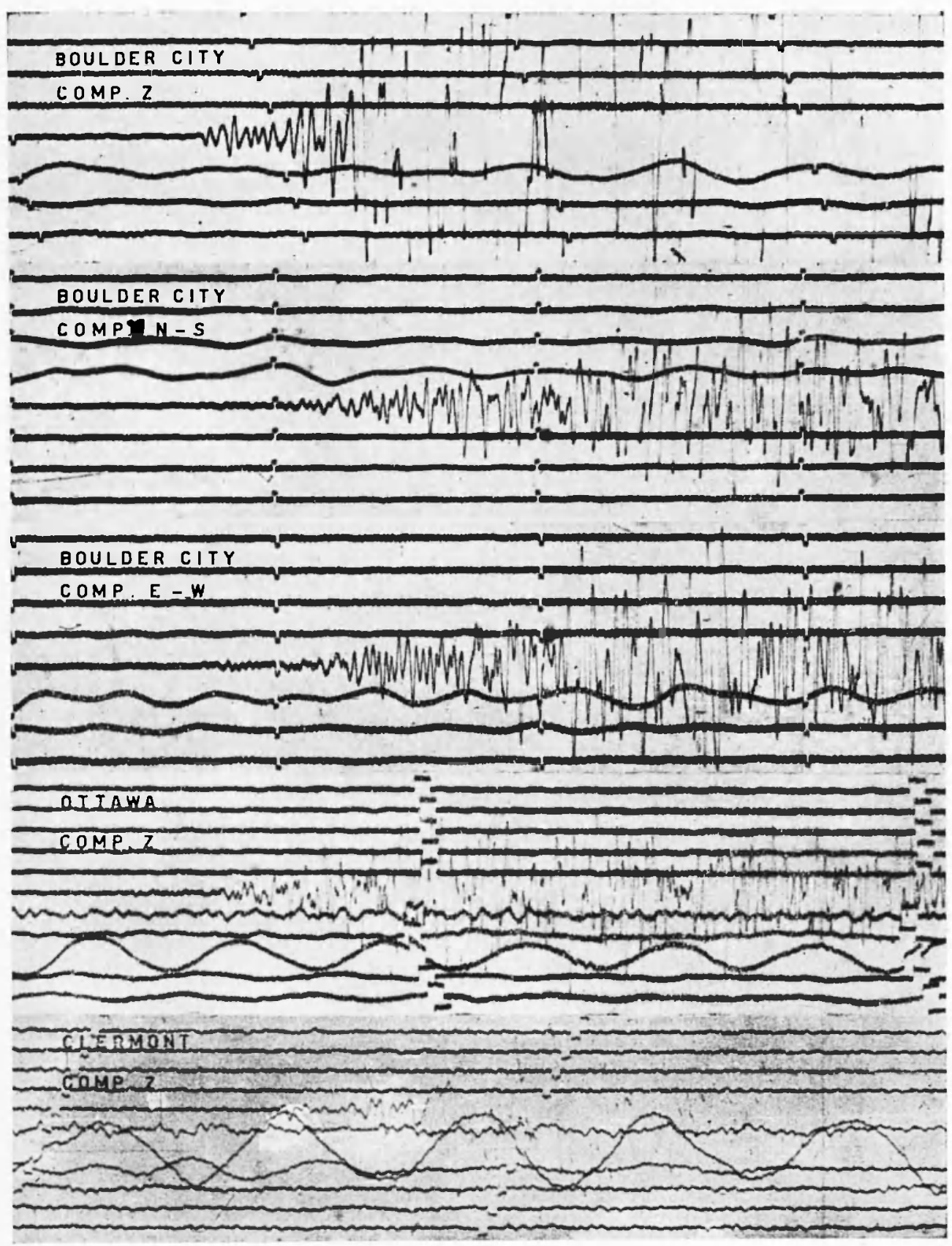

Fig. 3 
Ho assunto come valori provvisori per una prima approssimazione i seguenti :

$$
\begin{aligned}
& \left(\lambda_{0}\right)=+143^{\circ} 30^{\prime} \\
& \left(\phi_{0}\right)=+42^{\circ} 14^{\prime} .8 \text { (latitudine geocentrica), } \\
& (h)=33 \mathrm{~km} . \\
& \left(t_{0}\right)=\quad 01^{1 \mathrm{H}} 22^{\mathrm{m}} 58^{\mathrm{s}} .596 .
\end{aligned}
$$

I dati necessari per il calcolo sono riassunti nella tabella III. La soluzione del sistema normale dedotto dalle 43 corrispondenti equazioni di condizione ha dato luogo ai seguenti valori più probabili delle incognite

$$
\begin{aligned}
& \delta \lambda_{0}=+00^{\circ} 00^{\prime}, 688 \\
& \delta \phi_{0}=-00^{\prime \prime} 07^{\prime} .556 \\
& \delta h=+0,0129566 R=82,119 \mathrm{~km} . \quad(R=6338 \mathrm{~km} .), \\
& \delta t_{0}=+9^{*}, 06
\end{aligned}
$$

con i corrispondenti errori nedi :

$$
\begin{aligned}
& m_{\delta \lambda_{\mathrm{o}}}=\div 5^{\prime}, 247, \\
& m_{\delta \phi \mathrm{o}}= \pm 5^{\prime}, 624, \\
& m_{\delta h}= \pm 0,0079375 \mathrm{i}= \pm 50,308 \mathrm{~km} . \\
& m_{\delta t}= \pm 5^{\mathrm{s}, 7} .
\end{aligned}
$$

Come controllo ho ottenuto i seguenti risultati:

$$
[\vee]=75,1892787, \quad[11.4]=75,1892705 .
$$

Ritenendo troppo forti le correzioni da apportare alla profondità e al tempo origine, ho proceduto ad una seconda approssimazione, escludendo dal calcolo i dati della stazione di Tortosa che damo luogo ad una differenza $0-C$ di tre secondi circa. Ho assunto come valori provvisori per questa seconda approssimazione i seguenti:

$$
\begin{aligned}
& \left(\lambda_{0}\right)=+143^{\circ} 30^{\prime}, \\
& \left(\phi_{0}\right)=+42^{\circ} 07^{\prime}, 091, \\
& (h)=33 \mathrm{~km}+0,01295566 R=115,119 \mathrm{~km} ., \\
& \left(t_{0}\right)=01^{\mathrm{h}} 23^{\mathrm{m}} 07^{\mathrm{s}}, 658 .
\end{aligned}
$$




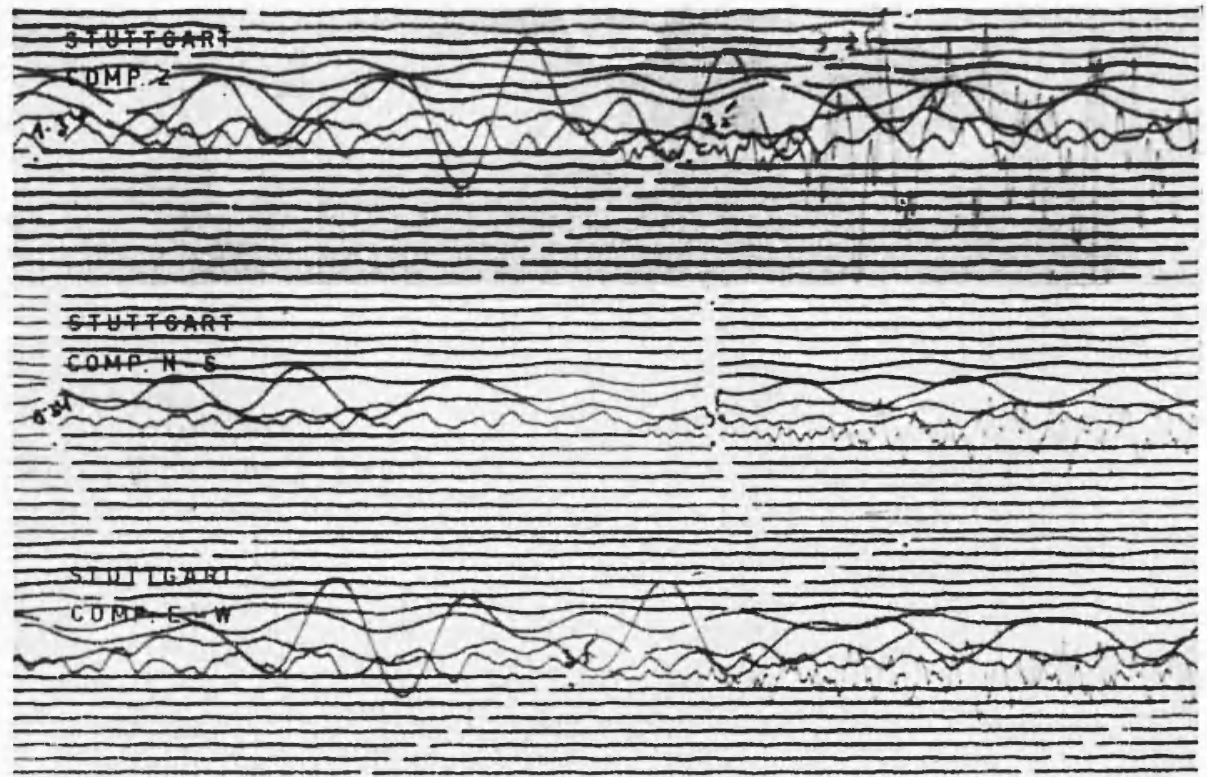

\section{PALISADES}

GOMP. Z

Whate

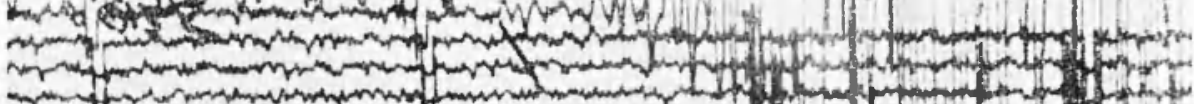

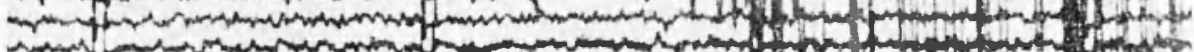

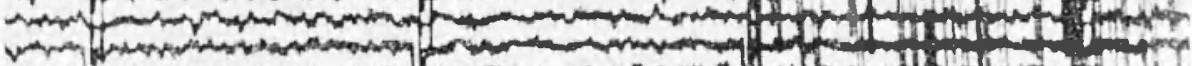

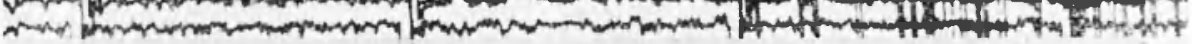

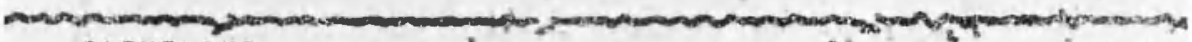
CLEVELAND

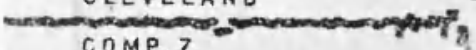

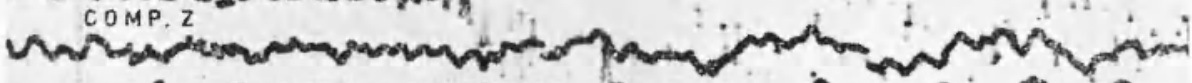

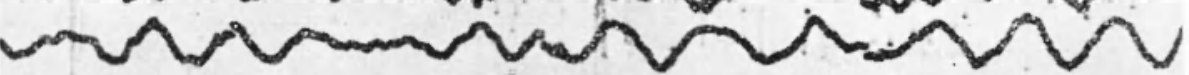

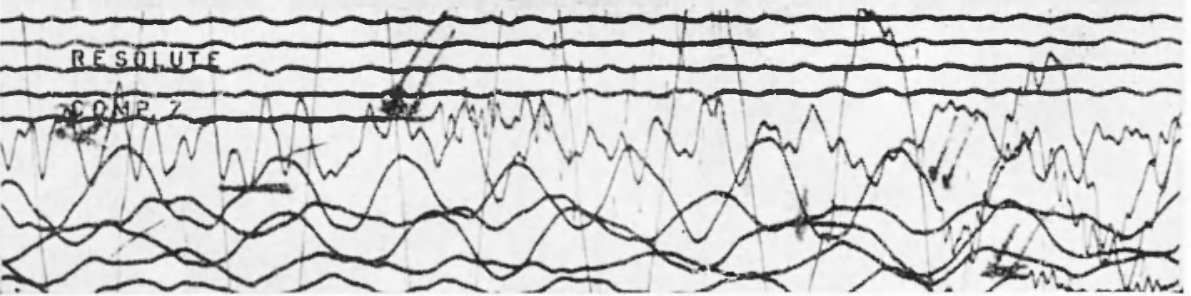

Fig. 4 


\begin{tabular}{|c|c|}
\hline$\frac{\stackrel{3}{3}}{1}$ & 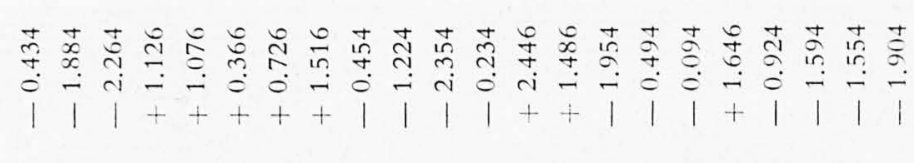 \\
\hline$\overline{0} \mid \overline{\frac{\Xi}{\sigma}}$ & 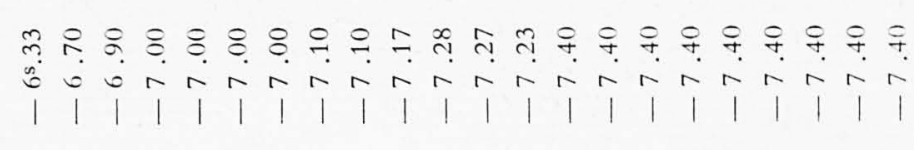 \\
\hline $\overrightarrow{0} \mid \widehat{c}$ & 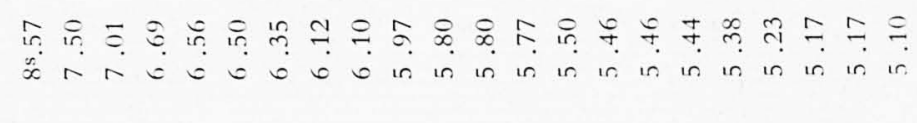 \\
\hline 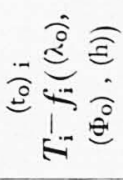 & 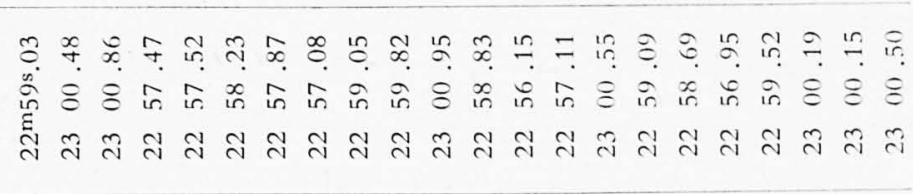 \\
\hline 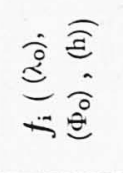 & 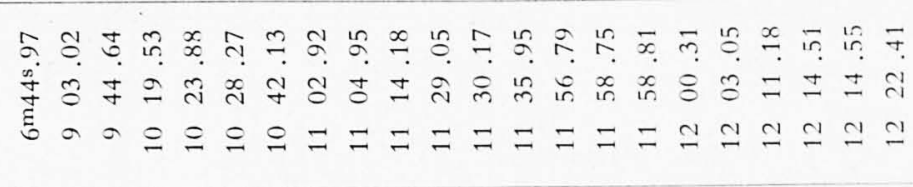 \\
\hline$\ddot{I}=$ & 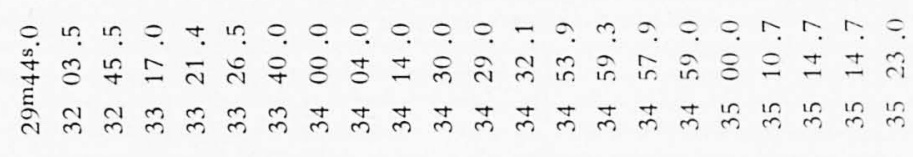 \\
\hline$\widehat{\mathrm{j}}$ & 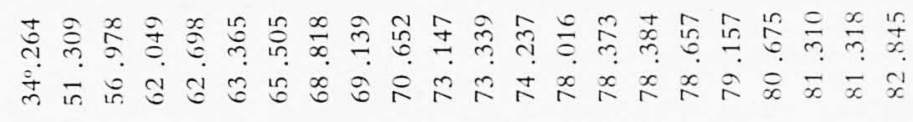 \\
\hline $\begin{array}{l}\vec{Z} \\
0 \\
\vec{N} \\
\ll \\
\text { is } \\
\text { is }\end{array}$ & 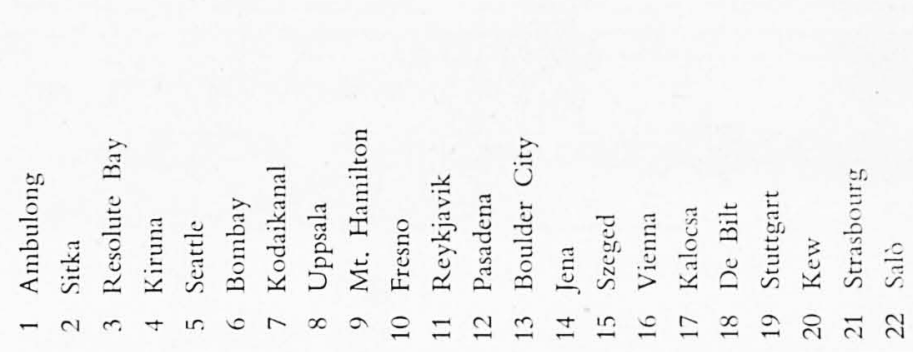 \\
\hline
\end{tabular}




\begin{tabular}{|c|c|}
\hline$\frac{3}{3}$ & 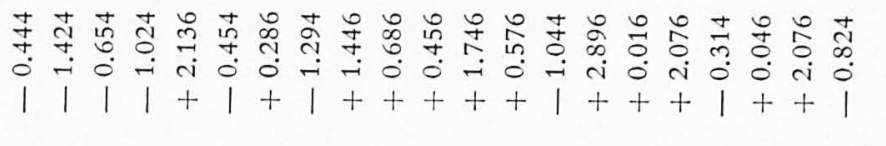 \\
\hline$\vec{\infty} \mid \stackrel{\equiv}{\infty}$ & 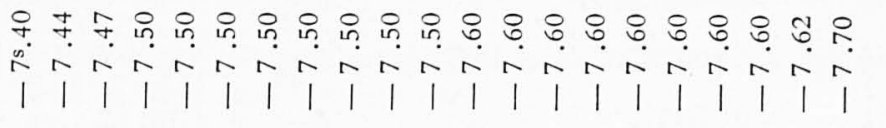 \\
\hline i) & 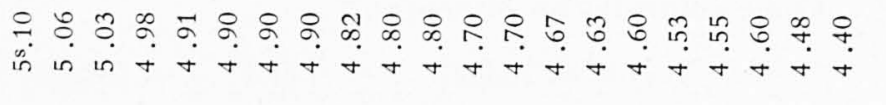 \\
\hline 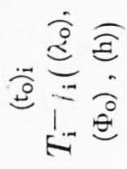 & 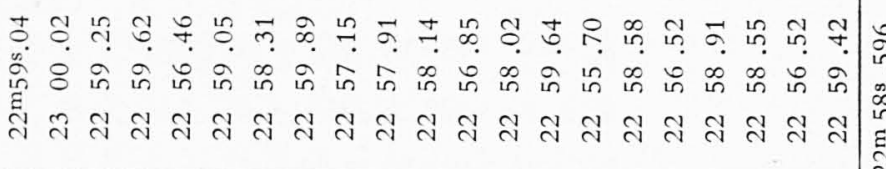 \\
\hline 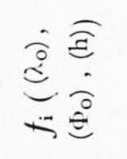 & 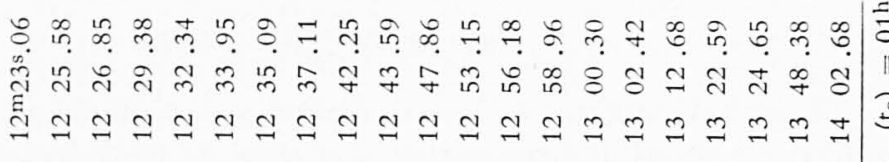 \\
\hline 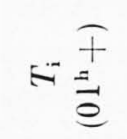 & 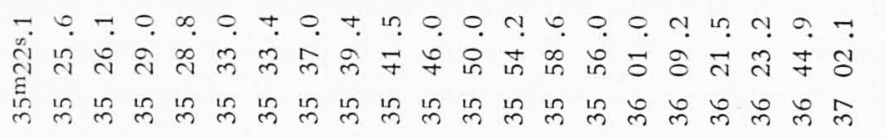 \\
\hline$\ddot{i}$ & 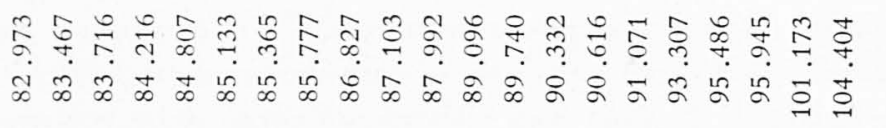 \\
\hline 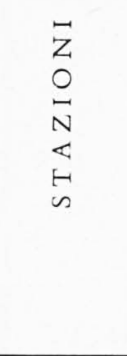 & 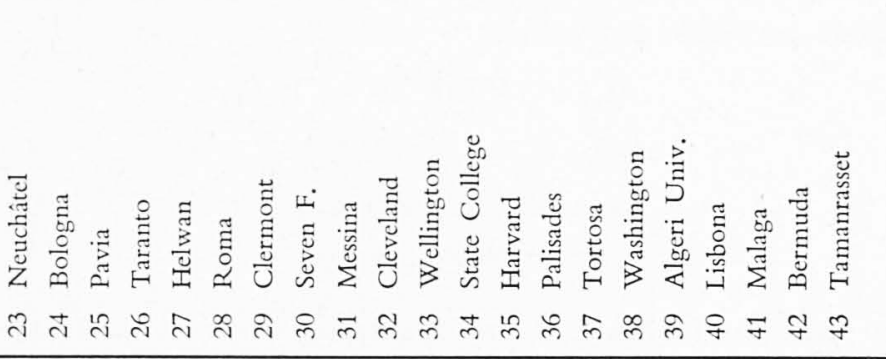 \\
\hline
\end{tabular}


Dedotti gli elementi necessari per il calcolo (riassunti nella tabella IV), la risoluzione del sistema delle 42 equazioni di condizione corrispondenti ha dato luogo ai seguenti risultati:

$$
\begin{array}{ll}
\delta \lambda_{\mathrm{o}}=+00^{\circ} 00^{\prime}, 373, & { }^{m_{\delta \lambda_{0}}}= \pm 00^{\circ} 05^{\prime}, 037, \\
\delta \phi_{0}=-00^{\circ} 00^{\prime}, 137, & m_{\delta_{\alpha}}= \pm 00^{\circ} 05^{\prime}, 541, \\
\delta h=-0,00073507 R=4,659 \mathrm{~km} ., & m_{\delta i_{1}}= \pm 0,0086 \mathrm{R}= \pm 54,507 \mathrm{~km} \cdot, \\
\delta t_{0}=-0^{*}, 537, & m_{\delta i_{0}}= \pm 6^{\circ}, 02 .
\end{array}
$$

Come controllo ho ottenuto:

$$
[\nu v]=68,163627, \quad[11.4]=68,163627 .
$$

Per le grandezze cercate si hanno pertanto i seguenti valori:

$$
\begin{aligned}
& \lambda_{0}=+143^{\circ} 30^{\prime}, 373 \div 05^{\prime}, 073, \\
& \Phi_{0}=+42^{\circ} 06^{\prime}, 95+1-05^{\prime}, 541, \\
& h=110,550 \div 54,507 \mathrm{~km} ., \\
& \mathrm{t}_{0}=01^{\mathrm{h}} 23^{\mathrm{m}} 07^{\mathrm{s}}, 121+6^{*}, 02 .
\end{aligned}
$$

I risultati ottenuti in questa ultima approssimazione, sebbene portino a correzioni di lieve entita, non sono da considerarsi migliori dei risultati conseguiti nei calcoli precedenti. Infatti, i limiti di incertezza entro i quali restano determinati la profondità e il tempo origine sono, in ambedue $\mathrm{i}$ casi, dello stesso ordine ed alquanto rilevanti. Oltre alle numerose cause di indeterminazione che generalmente influenzano l'approssimazione con cui si può determinare la posizione di un ipocentro $\left({ }^{6}\right)$, nel caso in esame notevole è il contriluto portato dai possibili errori di rilevamento, in considerazione del fatto che molti inizi relativi alle $P_{.2}$ sono stati rilevati nel corpo delle registrazioni. Comunque, eliminando dal calcolo i dati di tutte quelle stazioni che dano luogo ad una differenza $0-C$ superiore a $l^{s}, 5$, si possono ottenere risultati più approssimati. Ho proceduto ad una ulteriore approssimazione partendo dai valori:

$$
\begin{aligned}
& \left(\lambda_{\mathrm{o}}\right)=+143^{0} 30^{\prime}, \\
& \left(\Phi_{\mathrm{o}}\right)=+42^{\mathrm{o}} 07^{\prime}, 091, \\
& (h)=115,119 \mathrm{~km}, \\
& \left(t_{\mathrm{o}}\right)=01^{\mathrm{\prime}_{1}} 23^{\mathrm{m}} 08^{\mathrm{s}}, 193,
\end{aligned}
$$

ed utilizzando i dati delle 28 stazioni elencate nella tabella V. 


\begin{tabular}{|c|c|}
\hline$\frac{3}{2}$ & 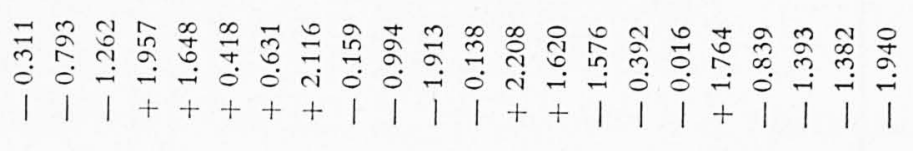 \\
\hline$\vec{\infty} \mid \underset{\infty}{\Xi}$ & 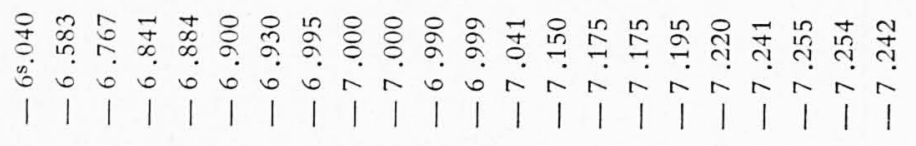 \\
\hline$\sqrt{\infty} \mid \widehat{\frac{1}{0}}$ & 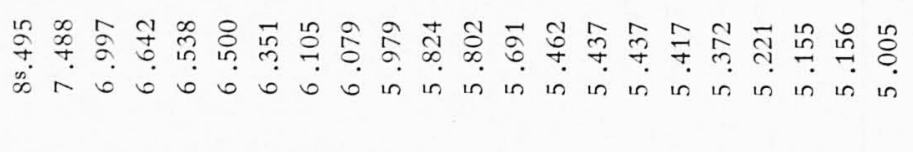 \\
\hline 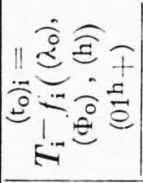 & 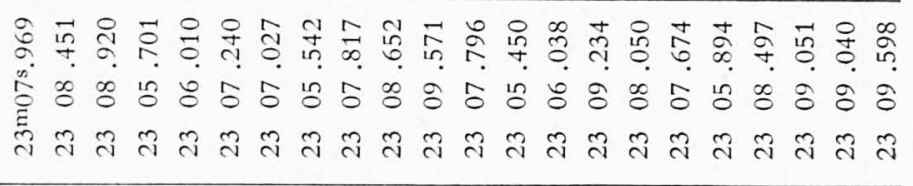 \\
\hline 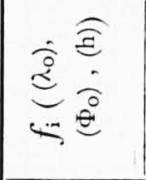 & 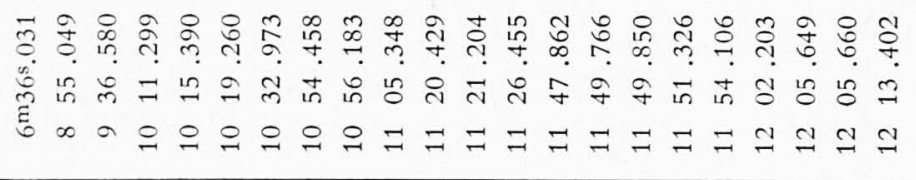 \\
\hline$\underset{\tilde{\theta}}{\tilde{E}}$ & 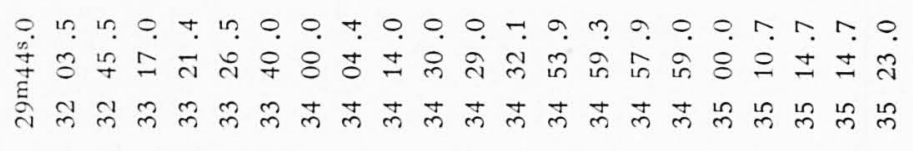 \\
\hline I & 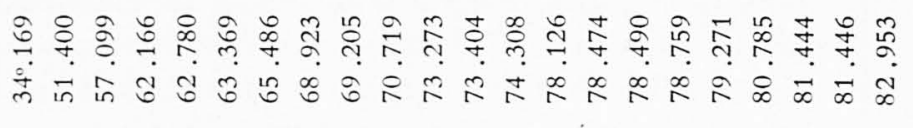 \\
\hline $\begin{array}{l}\vec{Z} \\
O \\
\stackrel{N}{N} \\
\ll \\
\qquad \\
\text { in }\end{array}$ & 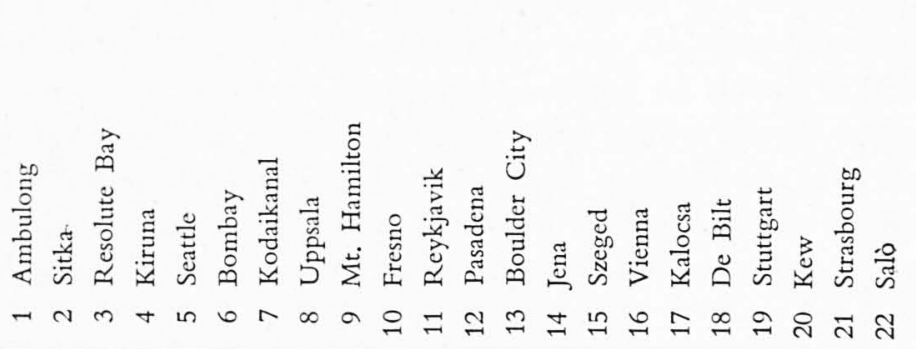 \\
\hline
\end{tabular}




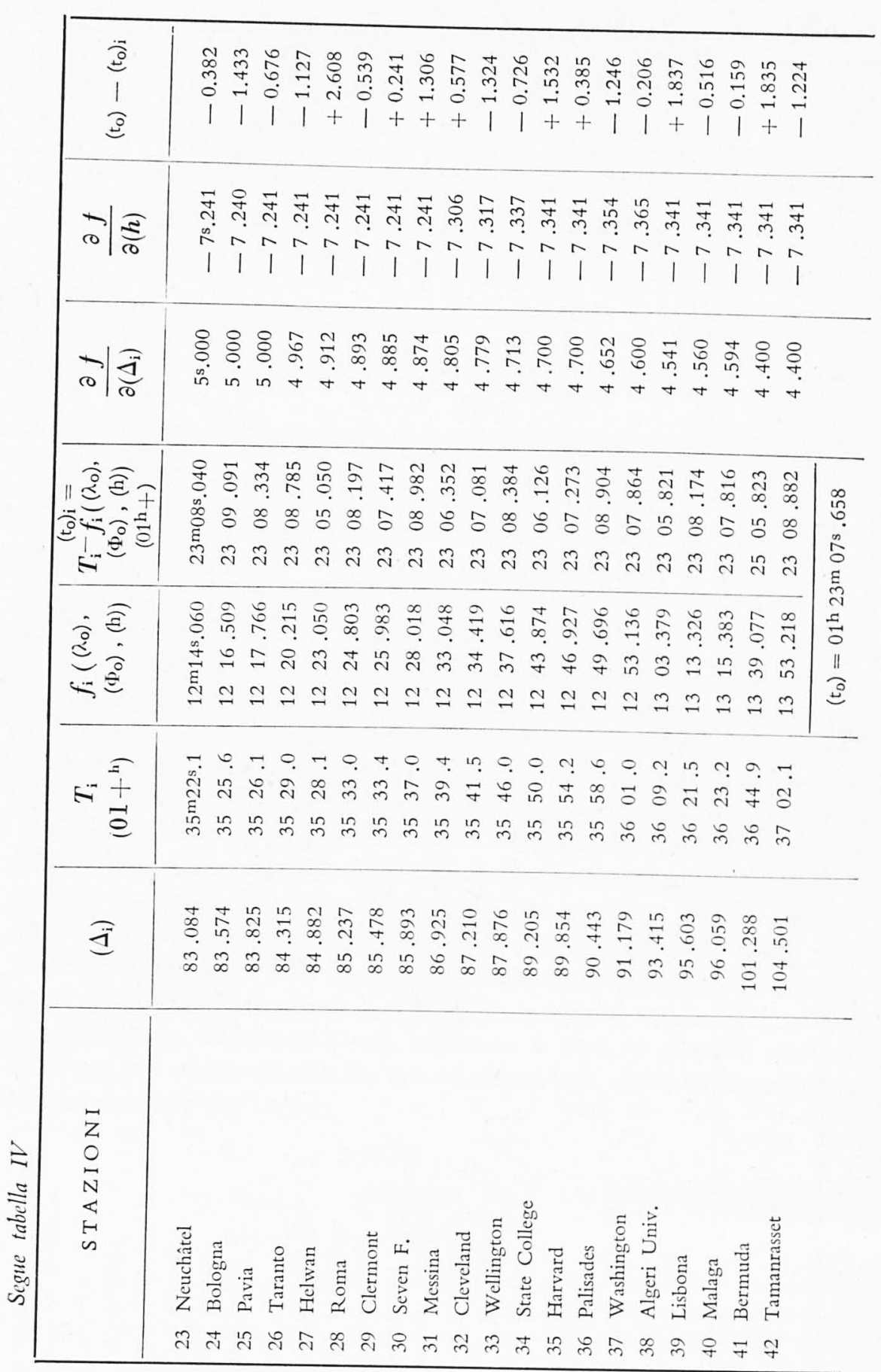


I risultati oftenuti sono $\mathrm{i}$ seguenti:

$$
\begin{aligned}
& \delta \lambda_{\mathrm{o}}=-00^{\circ} 00^{\prime}, 745, \quad m_{\delta \lambda_{0}}= \pm 00^{\prime \prime} 02^{\prime}, 884 ， \\
& \delta \Phi_{0}=-00^{\mathrm{n}} 03^{\prime}, 815, \quad \boldsymbol{m}_{\delta_{\phi 0}}= \pm 00^{\mathrm{n}} 02^{\prime}, 998, \\
& \delta h=+0,00091623 R=+5,807 \mathrm{~km}, m_{\delta h}=+0,004677 R=+29,643 \mathrm{~km} \text {. } \\
& \delta t_{o}=+0^{\circ}, 45, \quad m_{\delta^{\prime}}= \pm 3^{\mathrm{s}}, 4, \\
& {[v]=10,15371, \quad[11.4]=10,15372,}
\end{aligned}
$$

per cui i valori definitivi, forniti da quest'ultima approssimazione, sono:

$$
\begin{aligned}
& \lambda_{o}=143^{\circ} 29^{\prime} 255+02^{\prime}, 884, \\
& \omega_{o}=+42^{\circ} 03^{\prime} 276 \pm 02^{\prime}, 998, \\
& h=120,9-1-29,6 \mathrm{~km}, \\
& \iota_{c}=01^{\mathrm{h}} 23^{\mathrm{m}} 08^{\mathrm{s}}, 6+3^{\mathrm{s}}, 4 .
\end{aligned}
$$

1 alcoli eseguiti, oltre che confermare il supposto carattere multiplo del terremoto dello Fokkaido del 4, marzo 1952, permettono di specificare che una prima scossa superficiale è stata sequita, 24* circa più tardi, da una seconda scossa, la cui origine, avente praticamente le stesse coordinate epicentrali, si trova ad una profonditi dell'ordine del centinaio di chilometri.

E assai frequente il caso in cui, nei sismogrammi relativi a terremoti lontani di grande intensiti, le $P_{n}$ si presentano con una lieve emersio sequita da oscillazioni più o meno regolari interrotte da bruschi impulsi susseguentisi ad intervalli di tempo indipendenti dalla distanza. A tale moltepliciti di impulsi difficilmente si potrebhe dare griustificazione se fosse realmente valida l'ipotesi semplificatrice secondo la quale un terremoto è dovuto allo scatto di compartimenti delle stratificazioni terrestri quale unico atto conclusivo di uno stato di tensione interna maturatosi in un periodo di tempo più o meno lungro. Secondo alcuni ricercatori la molteplicita degli impulsi nelle registrazioni trova giustificazione nella molteplicita dei movimenti nella zona ipocentrale, nel senso che il dislocamento dei compartimenti della crosta, con cui si manifesta il terremoto, non è né isolato né improviso, ma si diffonde in un intervallo di tempo finito dando luogo a movimenti distinti. Secondo altri il fatto i presumibilmente da attribuirsi ad un particolare meccanismo di produzione all ipocentro che da luogo ad un processo, di una certa durata, nel corso del quale 
TABella $\mathrm{V}$

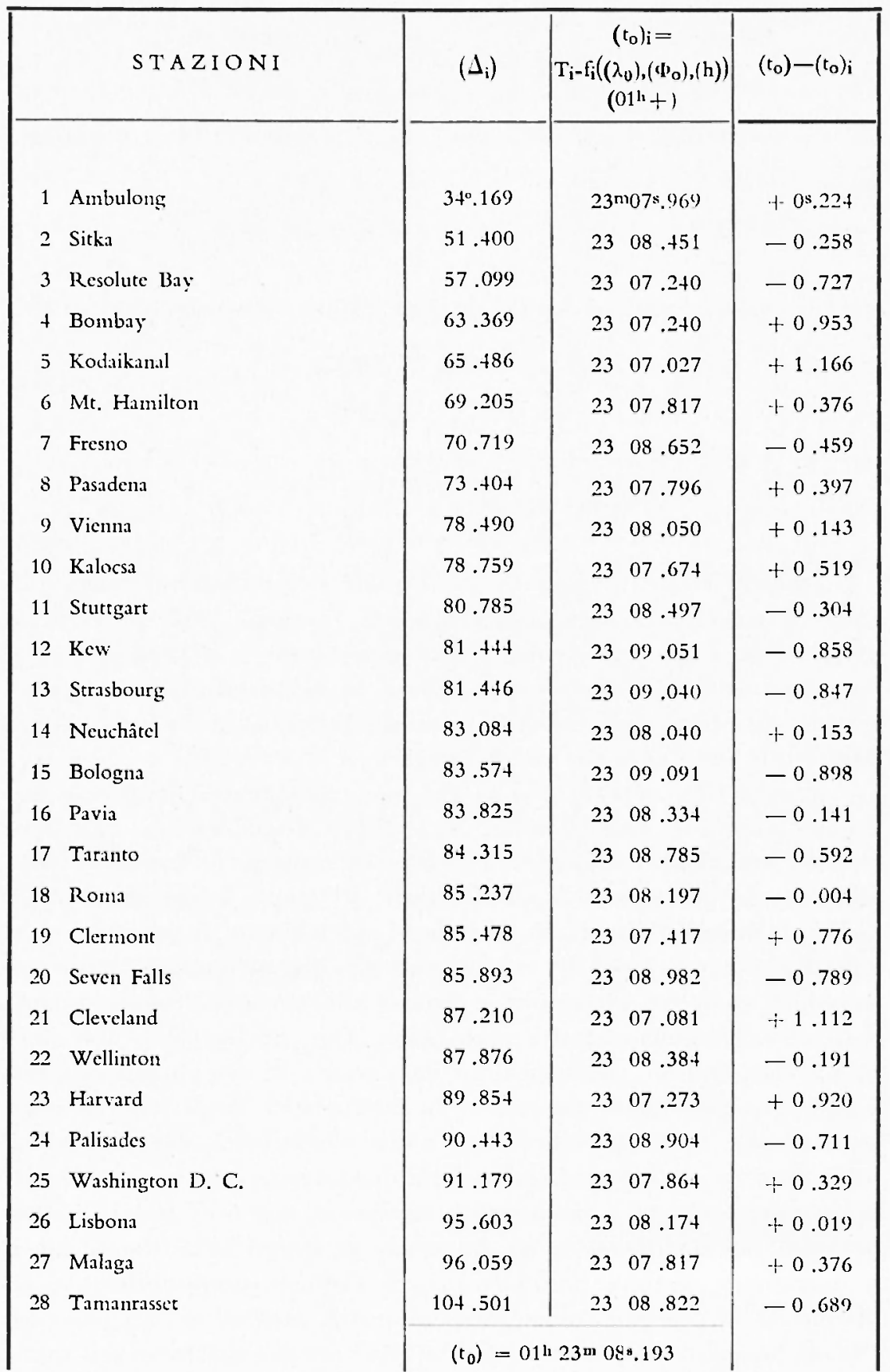


le vilurazioni a breve periodo possono venire modificate da fenomeni di interferenza. In dette ipotesi è implicita l'interdipendenza dei singoli processi cinematici e dinamici che, nel loro complesso costituiscono il fenomeno globale. La questione, riassunta nei termini sopra accennati, riveste un carattere generale ed è intimamente legata al complicato mecanismo che genera il fenomeno sismico. Nel caso preso in esame in questa nota, non vi sono elementi sufficienti per provare l'interdipendenza dei processi che hanno dato luogo alle due scosse. Non $\dot{i}$, infatti, agevole spiegarsi come una scossa superficiale ablia potuto stimolarne una seconda più intensa ad una profondità di cento chilometri. Si potreble pensare ad un unico processo maturatosi in profondita che ha dato luogo ad una manifestazione preliminare in superficie.

Istituto Nazionale di Geofisica - Osserv. di Messina - Settembre 1954.

\section{RIASSUNTO}

In seguito ad alcune caratteristiche riscontrate nei sismogrammi, sorge il sospetto che il terremoto dello Hokkaido del 4 marzo 1952 abbia lipocentro ad una profondità superiore alla normale, in contrasto con $i$ risultati, ottenuti in un lavoro precedente, che escludono la possibilità di una profondità apprezzabile in ottimo accordo con gli inizi delle $\mathrm{P}_{\mathrm{n}}$ osservati in un gruppo di 45 stazioni.

Viene notata una frequenza anomala di residui delle $\mathrm{P}_{\mathrm{n}}$ di $12^{s}$ circa. Questa circostanza, in aggiunta al fatto che un notevole numero di stazioni presenta una $\mathrm{i}\left(\mathrm{P}_{2}\right)$ circa $12^{s}$ dopo la registrazione della $\mathrm{P}$ $\left(\mathrm{P}_{1},{ }^{\prime}\right.$, il cui inizio è generalmente debole, induce a pensare che, $12^{\text {s }}$ circa dopo la registrazione di una prima scossa, le stazioni abbiano registrato una seconda scossa più intensa proveniente dalla stessa zona 'picentrale.

Vengono determinate le coordinate spazio-temporali della seconda scossa, adoperando un metodo statistico di Caloi. I calcoli, oltre che confermare il supposto carattere di scossa doppia del terremoto qui studiato, permettono di specificare che una prima scossa superficiale è stata seguita, $24^{s}$ circa più tardi, da una seconda scossa, la cui origine, avente praticamente le stesse coordinate epicentrali, si trova ad una profondità dellordine del centinaio di chilometri. 


\section{SUMMARY}

As a consequence of some characteristics encounted in the sismograms, the suspicion has arisen that the earthqualie of Holikaido of March 41952 has a focus and a depth greater than normal, in contrast with the results, of a previous work, which exclude the possibility of an apreciable depth in very good agreement with the beginnings of the $\mathrm{P}_{\mathrm{n}}$ observed in a group of 45 stations.

An anomalous frequency has been noticed in the residuals of the $\mathrm{P}_{\mathrm{n}}$ of about 12*. This circumstance, together with the fact that a noticeable number of stations present an $\mathrm{i}\left(\mathrm{P}_{2}\right)$ about $12^{*}$ after the registration of the $\mathrm{P}\left(\mathrm{P}_{1}\right)$, whose beginning is generally weak, leads one to think that, about $12^{s}$ after the registration of the first shock, the stations have registered a second, more intense shock coming from the same epicentral zone.

The space-time coordinates of the second quake have been determined, adopting a statistical mothod due to Caloi. The calculations, besides confirming the supposed double character of the earthqualie here studied, allow one to specify that a first superficial shock has been followed. about $24^{*}$ later, by a second shock, uhose origin, having pratically the same epicentral coordinates, has a depth on the order of 100 kilometers.

\section{BIBIIOGRAFIA}

(1) Ginutsoa A, $1 /$ terremoto dello Hohtiaido del 4 marzo 1952. "Annali di Geofisica ", vol. VI, n. 2, 1953.

(2) Girlanda A., Il terremoto dello Hokkaido del 4 marzo 1952. Parte II. "Annali di Geofisica ", vol. VI, n, 4, 1953.

(3) Jeffrers H. - Bullex K. E., Seismological Tables. British Association for the Advancement of Science: Gray Milne Trust, London 1910.

(4) Stonesey R., Tuo Double Earthquakes. Royal Astronomical Society. "Geophysic Supplement ", vol. 4, n. 6, 1939.

(5) Caloi P. - Perosaci F, $l l$ terremoto del Turkestom del 2 notembre 1930. Annali di Geofisica, vol. I, n, 2, 1918.

(6) Valle P. E., Sulla determinazione delle coordinate ipocentrili di un sisma lontano. "Bollettino dellal Società Sismologica Italianta ". wol. XL. n. 3-1, 19.43. 